\title{
Presentación de un caso de síndrome de Mauriac Reporte de caso clínico
}

\author{
Yessica Agudelo Zapata ${ }^{1}$, Camilo Andrés Quintero Cadavid ${ }^{1}$ Héctor Fabio Sandoval Alzate ${ }^{1}$, \\ Luis Miguel Maldonado', Roberto Franco Vega ${ }^{3}$.
}

${ }^{1}$ Residente Especialidad en Endocrinología. Departamento de Medicina Interna, Facultad de Medicina. Universidad Nacional de Colombia. Bogotá, D.C.

${ }^{2}$ Médico especialista en Endocrinología. Profesor Asociado. Departamento de Medicina Interna, Facultad de Medicina. Universidad Nacional de Colombia. Bogotá, D.C. ${ }^{3}$ Médico especialista en Endocrinología. Profesor Titular. Departamento de Medicina Interna, Facultad de Medicina. Universidad Nacional de Colombia. Bogotá, D.C.

Fecha de recepción: 15/06/2016

Fecha de aceptación: 30/06/2016

\section{Resumen}

S e presenta un paciente con diabetes mellitus tipo 1, con una complicación poco frecuente conocida como síndrome de Mauriac. Se realizan ayudas diagnósticas tendientes a descartar diagnósticos diferenciales como la mucopolisacaridosis tipo I, que se consideró una de las enfermedades de depósito más probables en el caso del paciente. Finalmente, se presenta una discusión del caso, resumiendo los aspectos fundamentales que llevaron a la sospecha del síndrome de Mauriac.

Palabras clave: diabetes mellitus tipo 1, enfermedades metabólicas, complicaciones de diabetes, deficiencia de lipasa ácida lisosomal, mucopolisacaridosis tipo 1, síndrome de Mauriac.

\section{Abstract}

This is a case report of a patient with diabetes mellitus type 1 and a rare complication known as Mauriac syndrome. Laboratory tests were performed to rule out differential diagnoses, such as mucopolysaccharidosis type I, which was considered one of the storage diseases, most likely for the patient. Finally, we present a discussion of the case, summarizing the key issues that led to the suspicion of a Mauriac syndrome.

\section{Introducción}

En un paciente con Diabetes Mellitus tipo 1 (DM1) la presencia concomitante de talla baja patológica, retraso puberal, hepatomegalia, alteraciones musculoesqueléticas y dislipidemia, hace sospechar una entidad clínica que es infrecuente y se asocia a mala adherencia al tratamiento, a dificultades para el acceso, oportunidad y calidad de los servicios de salud, entidad que es conocida como el síndrome de Mauriac, una enfermedad de depósito de glucógeno que ocurre como parte de un mal control metabólico a largo plazo del paciente con DM1.

El objetivo de presentar este caso clínico es mostrar a la comunidad médica, no sólo cómo sospechar el síndrome de Mauriac, sino evidenciar que este síndrome clínico es prevenible por medio del mejoramiento continuo de la atención en salud en diabetes.

\section{Presentación del caso}

Paciente de sexo masculino de 16 años 9 meses con antecedente de DM1 desde el año y medio de vida, en manejo con insulina basal-bolo. Durante el interrogatorio se evidencia que durante toda la infancia el paciente presenta dificultad para el acceso a servicios de salud, a los medicamentos, inadecuada adherencia y control metabólico. Convive con su madre y pertenecen a un estrato socioeconómico bajo, sin antecedentes perinatales de importancia. Actualmente, cursa bachillerato con rendimiento escolar promedio. Al momento de la consulta estaba en manejo con insulina glargina 28 unidades una vez al día, insulina glulisina 2-5-2 unidades preprandiales, captopril $12,5 \mathrm{mg}$ al día, lovastatina $20 \mathrm{mg}$ al día. Como antecedentes patológicos asociados tiene diagnóstico de dislipidemia mixta, talla baja y hepatomegalia que estaba en estudio en otra institución. A la revisión por sistemas sólo se encontraron síntomas de hipoglucemia ocasionales.

No tiene otros antecedentes personales relevantes. Dentro de los antecedentes familiares tiene un hermano con DM1 en terapia con bomba de insulina, actualmente de 22 años de edad y una hermana que falleció a los 4 años de edad sin otra información al respecto.

Al examen físico se encuentran signos vitales normales, peso: $34 \mathrm{~kg}$ Talla: $130 \mathrm{~cm}$ (DS -5,96) índice de masa corporal de $20,1 \mathrm{~kg} / \mathrm{m}^{2}$ (DE -0,44), envergadura $132 \mathrm{~cm}$, segmentos corporales superior e inferior simétricos. Tiene una frente prominente, cejas pobladas, tendencia a sinofris, nariz proyectada, filtrum largo, pestañas escasas, pabellones auriculares 


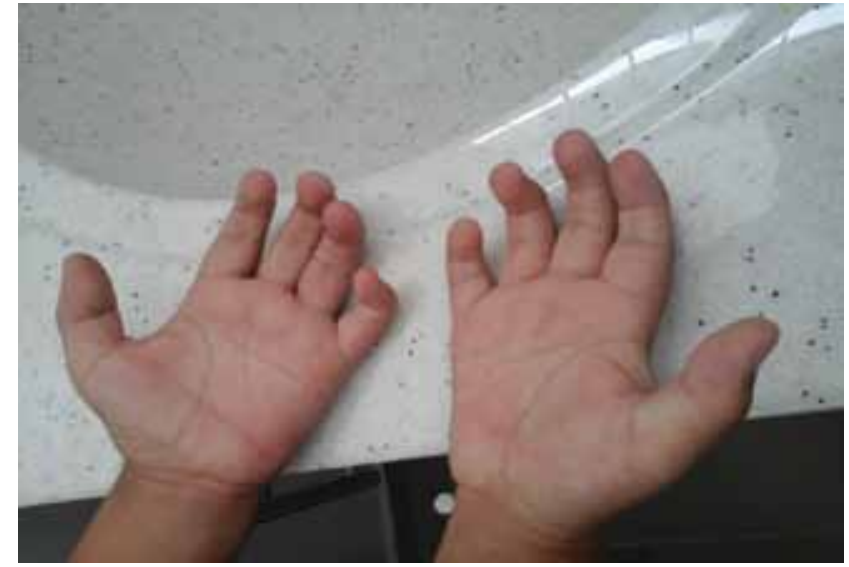

Figura 1. Hallazgo al examen físico: Manos en Garra

Figura 2. Examen físico: Abdomen globoso y hepatomegalia



Tabla 1. Pruebas hormonales

\begin{tabular}{l|c|c}
\hline \multicolumn{1}{c|}{ Prueba/fecha } & Un año antes & Fecha de consulta \\
\hline FSH & $0,58 \mathrm{mUl} / \mathrm{mL}$ & $1,06 \mathrm{mUl} / \mathrm{mL}(1,3-11,8)$ \\
\hline LH & $0,20 \mathrm{mUl} / \mathrm{mL}$ & $1,8 \mathrm{mUl} / \mathrm{mL}(2,8-6,8)$ \\
\hline Prolactina & $4,3 \mathrm{ng} / \mathrm{mL}$ & $21 \mathrm{ng} / \mathrm{mL}$ \\
\hline Testosterona total & $0,16 \mathrm{ng} / \mathrm{mL}$ & $0,52 \mathrm{ng} / \mathrm{mL}(0,28-11)$ \\
\hline T4L & & $1,32 \mathrm{ng} / \mathrm{dL}(0,8-1,7)$ \\
\hline TSH & & $3,31 \mathrm{mcUl} / \mathrm{mL}(0,3-3,6)$ \\
\hline Cortisol basal & & $9,8 \mathrm{mcg} / \mathrm{dL}$ \\
\hline Somatomedina C & & $112 \mathrm{ng} / \mathrm{mL}(244-511 \mathrm{por}$ Tanner $)$ \\
\hline Ca total & & $1,28 \mathrm{mmol} / \mathrm{L}$ \\
\hline $\begin{array}{l}\text { Relación Alb/Cr en orina } \\
\text { Ocasional }\end{array}$ & & $8,4 \mathrm{y} 13 \mathrm{mg} / \mathrm{g}$ \\
\hline Cortisol urinario & & $84,2 \mathrm{mcg} / 24$ horas \\
\hline
\end{tabular}

FHS: Hormona Folículo Estimulante, LH: Hormona Luteinizante, TSH: Hormona Estimulante del Tiroi des, Ca: calcio, Alb: albúmina, Cr: creatinina normo-implantados. La evaluación cardiopulmonar es normal. El abdomen es prominente, blando, depresible, no doloroso a la palpación, con hígado palpable hasta $3 \mathrm{~cm}$ por debajo del reborde costal derecho. Tiene genitales masculinos normales con volumen testicular de $12 \mathrm{~cm}^{3}$, pene con Tanner de 3 y vello púbico de 2. En las extremidades llama la atención contractura en flexión de articulación interfalángica distal de todos los dedos de ambas manos, con limitación funcional (figura 1 y 2). Además, presenta ligera limitación para la extensión de ambos codos. Al examen neurológico no se evidencian alteraciones.

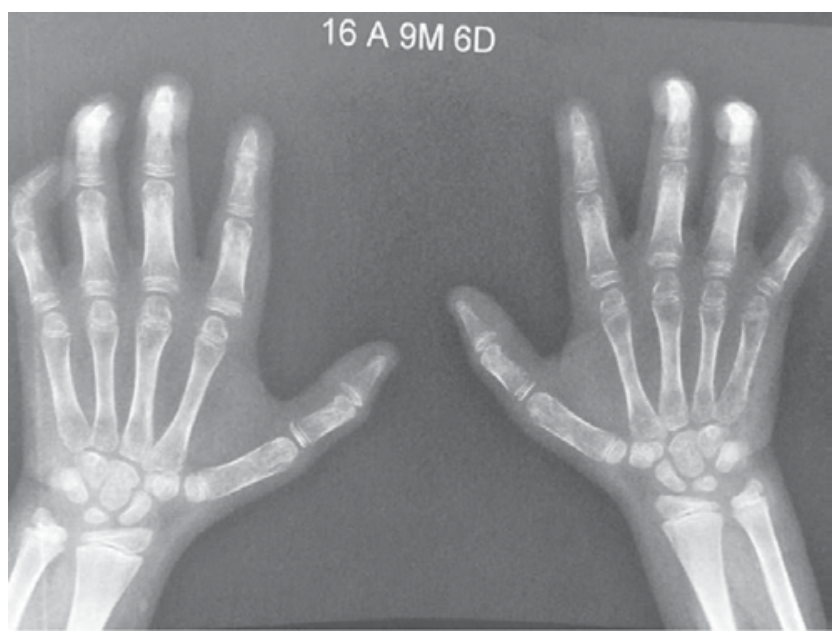

Figura 3. Carpograma

En los laboratorios del año anterior (tabla 1) se encontraba inactividad del eje gonadal con posterior corroboración bioquímica de inicio de pubertad. Presentaba además niveles de somatomedina $\mathrm{C}$ por debajo del valor de referencia para el estadio de Tanner en el momento de su realización. Se descartó alteración del eje tiroideo. Resonancia magnética nuclear de silla turca sin alteraciones. El carpograma (figura 3) del mes previo evidenciaba un retraso importante en la maduración con una edad ósea de 10 años. En la ecografía abdominal describen un aumento difuso de la ecogenicidad (interpretado como hígado graso) y un diámetro longitudinal de 18 cm (hepatomegalia) sin esplenomegalia ni ascitis. El perfil hepático (tabla 2) mostraba elevación de aminotransferasas y fosfatasa alcalina sin evidencia de alteración funcional ni explicación infecciosa o autoinmune de las alteraciones descritas, mientras que en el perfil lipídico (tabla 3) se encontró dislipidemia mixta con colesterol de HDL bajo. En laboratorios solicitados (tablas 4 y 5) tenía valores de hemoglobina glucosilada (HbA1c) que sugerían mal control glucémico. En los rayos $\mathrm{X}$ de columna y de huesos largos no se encontraron alteraciones. 
Tabla 2. Perfil hepático

\begin{tabular}{l|c}
\multicolumn{1}{c|}{ Prueba } & Fecha de consulta \\
\hline Aspartato aminotransferasa & $108 \mathrm{U} / \mathrm{L}$ \\
\hline Alanino aminotransferasa & $94 \mathrm{U} / \mathrm{L}$ \\
\hline Fosfatasa alcalina & $256 \mathrm{mg} / \mathrm{dL}$ \\
\hline Albúmina & $4,2 \mathrm{~g} / \mathrm{dL}$ \\
\hline $\begin{array}{l}\text { Anticuerpos anti- } \\
\text { mitocondriales }\end{array}$ & Negativos \\
\hline $\begin{array}{l}\text { Anticuerpos anti-músculo } \\
\text { liso }\end{array}$ & $1: 20$ Negativo \\
\hline $\begin{array}{l}\text { Anticuerpos antinucleares } \\
\text { (positivo mayor o igual a 1:80) }\end{array}$ \\
\hline $\begin{array}{l}\text { Antígeno de superficie de } \\
\text { hepatitis B } \\
\begin{array}{l}\text { Anticuerpos contra virus de } \\
\text { la hepatitis C }\end{array}\end{array}$ \\
\hline $\begin{array}{l}\text { Anticuerpos IgG contra virus } \\
\text { de la hepatitis A }\end{array}$ & Negativos \\
\hline
\end{tabular}

Tabla 3. Perfil metabólico

\begin{tabular}{l|c|c}
\hline Perfil metabólico & Un año antes & Fecha de consulta \\
\hline Colesterol de LDL & $166 \mathrm{mg} / \mathrm{dl}$ & $184 \mathrm{mg} / \mathrm{dl}$ \\
\hline Colesterol de HDL & $32 \mathrm{mg} / \mathrm{dl}$ & $29 \mathrm{mg} / \mathrm{dl}$ \\
\hline Colesterol TOTAL & $254 \mathrm{mg} / \mathrm{dl}$ & $274 \mathrm{mg} / \mathrm{dl}$ \\
\hline Triglicéridos & $377 \mathrm{mg} / \mathrm{dl}$ & $303 \mathrm{mg} / \mathrm{dl}$ \\
\hline HbA1c & $11 \%$ & $9,8 \%$ \\
\hline
\end{tabular}

LDL: lipoproteína de baja densidad, HDL: lipoproteína de alta densidad HbA1c: Hemoglobina glicosilada.

Tabla 4. Otros

\begin{tabular}{l|c}
\multicolumn{1}{c|}{ Prueba } & Fecha de consulta \\
\hline Leucocitos & $7.500 / \mathrm{mm}^{3}$ \\
\hline Neutrófilos \% & $59 \%$ \\
\hline Linfocitos \% & $33 \%$ \\
\hline Hemoglobina & $14,2 \mathrm{~g} / \mathrm{dl}$ \\
\hline Hematocrito & $42,4 \%$ \\
\hline Plaquetas & $556.000 / \mathrm{mm}^{3}$ \\
\hline Sodio & $136 \mathrm{mEq} / \mathrm{L}$ \\
\hline Potasio & $4,88 \mathrm{mEq} / \mathrm{L}$ \\
\hline Cloro & $99,8 \mathrm{mEq} / \mathrm{L}$ \\
\hline Creatinina & $0,6 \mathrm{mg} / \mathrm{dl}$ \\
\hline Gases arteriales & $\mathrm{PH} 7,4 \mathrm{pcO}_{2} 26,2 \mathrm{pO} 75,3 \mathrm{HCO}_{3} 19,1$ \\
\hline
\end{tabular}

\section{Discusión}

Este caso clínico ilustra el enfoque de un paciente con DM1 y sospecha de síndrome de Mauriac. El análisis que realizó el servicio de Endocrinología de la Universidad Nacional de Colombia fue sindromático. Si bien los pacientes con DM1 pueden presentar como parte de la historia natural de la enfermedad, talla baja y retraso puberal, había hallazgos adicionales al examen físico y al laboratorio que llevaron a configurar la sospecha diagnóstica de síndrome de Mauriac y fue la siguiente triada de estudio: hepatomegalia con estudio de virus hepatotropos y de autoinmunidad negativos, abriendo la posibilidad diagnóstica de una enfermedad de depósito, como puede ser la de glucógeno en contexto del paciente con DM1 y como lo sugiere el enfoque del paciente pediátrico con hepatomegalia ${ }^{(1)}$; dislipidemia (mixta con LDL elevado); y manos en garra (figura 1).

Este síndrome descrito por Mauriac en 1930, con algunos reportes en la literatura y siendo más frecuente en países en vías de desarrollo, se caracteriza por niños prepúberes con hepatomegalia, con aumento de las transaminasas, compromiso del crecimiento, retraso puberal y características cushingoides en contexto de una DM1 pobremente controlada ${ }^{(2)}$. En adultos jóvenes, el síndrome se presenta usualmente incompleto y sólo se encuentran hepatomegalia y aumento de las transaminasas; en pacientes de mayor edad, fácilmente se puede confundir con un hígado graso no alcohólico que es más frecuente en la diabetes mellitus tipo $2^{(3)}$. Esta glucogenosis hepática (enfermedad por depósito de glucógeno) se encuentra en el grupo de las enfermedades por depósito secundarias, ya que las primarias son enfermedades hereditarias que afectan a músculo e hígado y que se caracterizan por hepatomegalia, hipoglucemia, debilidad muscular y retraso del crecimiento ${ }^{(4)}$. En pacientes con DM1 y pobre control glucémico, dos eventos combinados están presentes para favorecer el depósito de glucógeno: la hiperglucemia y la cantidad (inadecuada) de dosis diaria de insulina. Durante la hiperglucemia se favorece la entrada de glucosa al hepatocito por medio de los receptores GLUT2 (glucose transporter 2, por sus siglas en inglés) (independientes de insulina) y esta es rápidamente fosforilada por lo que queda "atrapada" al interior del hepatocito. La administración de insulina a dosis fluctuantes permite en algunos momentos, por dosis insuficiente, la presencia de hiperglucemia y en otros momentos por dosis inadecuadamente altas, la promoción de la glucogenogénesis, con un balance neto hacia el depósito de glucógeno hepático que se traduce en hepatomegalia y elevación de transaminasas $^{(3)}$. El retraso del crecimiento está menos entendido y se especula que se debe a una homeostasis inadecuada de energía, una reducción en los niveles circulantes de IGF-1 y un estado de "resistencia" al efecto de la hormona de crecimiento (por alteración de la funcionalidad del receptor de IGF-1), sumado a un estado de hipercortisolismo como consecuencia de los episodios 
Tabla 5. Pruebas enzimáticas y moleculares

\begin{tabular}{l|c|c}
\multicolumn{1}{c|}{ Prueba } & Resultado & Control normal \\
\hline Actividad de lipasa ácida lisosomal & 858 (Negativo) & $40-600 \mathrm{pmol} / \mathrm{h} / \mathrm{spt}$ \\
\hline Alfa-L-iduronidasa (GSSPF) & $\begin{array}{c}13,65 \\
\text { (Negativo) }\end{array}$ & $1,5-20,1 \mathrm{nmol} / \mathrm{ml} / \mathrm{hora}$ \\
\hline Arilsulfatasa B (GSSPF) & $10,47(\mathrm{Negativo})$ & $2,9-43,2 \mathrm{nmol} / \mathrm{ml} / \mathrm{hora}$ \\
\hline Alfa-L-iduronidasa leucocitos & $\begin{array}{c}6,82 \\
(\text { Negativo) }\end{array}$ & $\begin{array}{c}4-16 \\
\mathrm{nmol} / \mathrm{mg} \mathrm{proteína/hora}\end{array}$ \\
\hline $\begin{array}{l}\text { Arilsulfatasa B } \\
\text { leucocitos }\end{array}$ & $\begin{array}{c}336,7 \\
(\text { Negativo) }\end{array}$ & $\begin{array}{c}115-226 \\
\mathrm{nmol} / \mathrm{mg} \mathrm{proteína/hora}\end{array}$ \\
\hline $\begin{array}{l}\text { Análisis genético molecular } \\
\text { por secuenciación para } \\
\text { mucopolisacaridosis tipo I (Gen IDUA) }\end{array}$ & $\begin{array}{l}\text { Se encontró una variante que fue clasificada como } \\
\text { benigna. Estos hallazgos no brindan evidencia molecular } \\
\text { que soporte el diagnóstico de mucopolisacaridosis tipo I. }\end{array}$ & \\
\hline
\end{tabular}

alternantes de hipoglucemia y $\operatorname{cetosis}^{(5,6)}$. Por las alteraciones hepáticas se deben considerar otras etiologías como las causas infecciosas, metabólicas (enfermedad de Wilson), neoplásicas y autoinmunes ${ }^{(3)}$, reportándose en algunos casos la realización de biopsia hepática con la confirmación histológica de depósito de glucógeno ${ }^{(7,8)}$. También debe tenerse en cuenta que usualmente no se reportan alteraciones en las pruebas de función hepática (albúmina, tiempo de protrombina). El tratamiento consiste en mejorar el control metabólico con la intensificación de la terapia insulínica, por lo que con la disponibilidad de los análogos de insulina y de las terapias con bombas de insulina se espera que ya no tengamos reportes de síndrome de Mauriac. Con el control metabólico se encuentra que hay regresión de la hepatomegalia, normalización de las transaminasas, mejoría del crecimiento, aunque sin lograr siempre la talla adulta final esperada ${ }^{(3,5,7,9)}$. Algunos casos reportan empeoramiento de las complicaciones microvasculares de la diabetes (retinopatía y nefropatía) con la intensificación muy agresiva de la terapia por lo que debe hacerse de manera progresiva ${ }^{(7)}$.

Otros diagnósticos diferenciales que se consideraron en este caso clínico fueron la deficiencia de lipasa ácida lisosomal y la mucopolisacaridosis tipo I, dado que al inicio la hepatomegalia había sido reportada con características ultrasonografías de hígado graso (que no eran claras), pero que junto con la elevación de transaminasas y la dislipidemia, hicieron sospechar el diagnóstico de deficiencia de lipasa ácida lisosomal ${ }^{(10)}$, por lo que se realizó prueba de actividad enzimática que fue negativa (tabla 5). Simultáneamente, la hepatomegalia y las manos en garra fueron consideradas por el servicio de genética y neuropediatría como sugestivas de mucopolisacaridosis tipo $1^{(11)}$, por lo que, para descartarlo se realizó test enzimático para iduronidasa, y pruebas moleculares que fueron negativas (tabla 5). Descartar otros diagnósticos diferenciales permitió reforzar la sospecha de síndrome de Mauriac.

El paciente actualmente se encuentra en seguimiento por el servicio de endocrinología con terapia basal-bolo prescrita, y ya se realizó trámite para iniciar terapia con bomba de insulina dada la presencia de una importante variabilidad glucémica. Se espera que con la mejoría del control glucémico haya mejoría de la hepatomegalia, la dislipidemia y de las contracturas musculoesqueléticas. No se consideró biopsia hepática, dada la reversión de los cambios demostrada en otros casos reportados en la literatura con la mejoría del control glucémico, por lo que quedan aspectos sin resolver aún en el caso y que el tiempo y la mejoría de control confirmarán la hipótesis diagnóstica.

El diagnóstico de síndrome de Mauriac evidencia las barreras en atención en salud del paciente con diagnóstico de DM1, desde la mala adherencia a los tratamientos hasta las dificultades para acceder a medicamentos y a la atención especializada oportuna, lo que convierte este síndrome clínico en prevenible. El mejoramiento continuo de los programas de atención al paciente con diabetes asegurará, en un futuro, que no haya más casos reportados de síndrome de Mauriac.

\section{Referencias}

1. Muñoz G. Hepatomegalia. Pediatr Integral. 2015; XIX (3): 180-197.

2. Mauriac P. Grosventre, hepatomegalie, troble de la croissance chez les enfants diabetiques: traits depuis plusieursannesparl'insuline. Gaz Hebl Sci Med Bordeaux 1930; 26: 402-410.

3. Giordano S, Martocchia A, Toussan L, Stefanelli M, Pastore F, Devito A, Risicato MG, Ruco L, Falaschi P. Diagnosis of hepatic glycogenosis in poorly controlled type 1 diabetes mellitus. World J Diabetes 2014; 5(6): 882-888.

4. Martocchia A, Risicato MG, Mattioli C, Antonelli M, Ruco L, Falaschi P. Association of diffuse liver glycogenosis and mild focal macrovesicular steatosis in a patient with poorly controlled type 1 diabetes. Intern Emerg Med 2008; 3: $273-274$.

5. Kim MS, Quintos JB. Mauriac syndrome: Growth failure and type 1 diabetes mellitus. Pediatric endocrinology reviews 2008; 5 suppl 4: 989-93.

6. Elder, C J. and A. Natarajan. "Mauriac Syndrome - A Modern Reality". Journal of Pediatric Endocrinology and Metabolism 2011; 23: 311-314.

7. Fitzpatrick E, Cotoi C, Quaglia A, et al. Hepatopathy of Mauriac syndrome: a retrospective review from a tertiary liver centre. Arch Dis Child 2014; 99:354-357.

8. Bua, Jenny Bussani, Rossana et al. Hepatic Glycogenosis in an Adolescent with Diabetes. The Journal of Pediatrics 2010;157(6): 1042.

9. Flotats Bastardas $\mathrm{M}$ et al. Hepatomegalia por depósito de glucógeno hepático y diabetes mellitus tipo 1. An Pediatr (Barc). 2007;67(2):157-60.

10. Guardamagna O, Nair D, Soran H, Hovingh K, Calandra S, Hamilton J, et al. Lysosomal acid lipase de ficiency e An under-recognized cause of dyslipidaemia and liver dysfunction. Atherosclerosis. 2014;235:21-30.

11. Rua M. Enfermedades metabólicas lisosomales manifestaciones osteoarticulares. Protoc diagn ter pediatr. 2014;1:231-9. 